\title{
Simulación de la microdeformación ósea de bruxismo en implante dental anterior
}

\author{
Simulation of bone microdeformation of bruxism in anterior \\ dental implant
}
Simulação de microdeformação óssea de bruxismo em implante dentário anterior

\author{
Ana María Sánchez Santamaría ${ }^{1}$ \\ Ana Catalina Castaño Posada ${ }^{2}$ \\ Federico Latorre Correa ${ }^{3}$ \\ Junes Abdul Villarraga Ossa ${ }^{4}$ \\ Juan Gustavo Diosa Peña ${ }^{5}$ \\ Samuel David Giraldo Gómez ${ }^{6}$
}

Recibido: 3 de febrero 2019

Aprobado: 27 de julio 2020

Publicado: 4 de septiembre de 2020

Cómo citar este artículo:

Sánchez-Santamaría AM, Castaño-Posada AC, Latorre-Correa F, Villarraga-Ossa JA, Diosa-Peña JG. Simulación de la microdeformación ósea de bruxismo en implante dental anterior. Revista Nacional de Odontología. (2020); 16(1), 1-18. doi: https://doi.org/10.16925/2357-4607.2020.01.12

Artículo de investigación. https://doi.org/10.16925/2357-4607.2020.01.12

1 Universidad Cooperativa, Medellín, Colombia.

ORCID: https://orcid.org/0000-0003-0470-4382

2 Universidad Cooperativa, Medellín, Colombia.

ORCID: https://orcid.org/0000-0003-2782-140X

3 Universidad de Antioquia, Medellín, Colombia. Odontólogo, Especialista en Prostodoncia, profesor titular, Facultad de Odontología, Grupo de investigación Biomat, Universidad de Antioquia, Medellín, Colombia. Dirección: Universidad de Antioquia. Facultad de Odontología. Calle 64 N$^{\circ}$ 52-59 Of. 107. Apartado Postal1226.

Correo electrónico: federico.latorre@udea.edu.co. ORCID: https://orcid.org/0000-0003-4432-3584

$4 \quad$ Universidad de Antioquia, Medellín, Colombia. ORCID: https://orcid.org/0000-0002-7893-5362

5 Universidad de Antioquia, Medellín, Colombia. ORCID: https://orcid.org/0000-0002-8064-2470

$6 \quad$ Universidad Cooperativa, Medellín, Colombia. ORCID: https://orcid.org/0000-0001-6645-8792 


\title{
Resumen
}

Introducción: el propósito de esta investigación fue evaluar la microdeformación ósea del bruxismo con implantes dentales por medio del método de análisis de elementos finitos (FEA).

Materiales y métodos: se modeló un (1) implante Tapered Screw-Vent ${ }^{\circledR}$ (ref. TSVB10 Zimmer Dental) de 13mm de longitud x $3.7 \mathrm{~mm}$ de diámetro con una plataforma de $3.5 \mathrm{~mm}$, un pilar en Zirconio, un tornillo, agente cementante cemento de resina, una corona cerámica monolítica de un incisivo central superior, hueso cortical y hueso esponjoso, utilizando el Software CAD de Solid Works 2010 (SolidWorks Corp., Concord, MA, USA), y posteriormente se procesó y se analizó a través del Software ANSYS versión 14. Se evaluaron los esfuerzos von Mises y microdeformación ósea ( $\mu$ strain), aplicando esfuerzos en sentido oblicuo con magnitudes de $200 \mathrm{~N}$ y $800 N$. Este análisis permitió evaluar y comparar la ( $\mu$ strain), tanto en el hueso cortical como en el hueso esponjoso en dos magnitudes $200 \mathrm{~N}$ Y $800 \mathrm{~N}$.

Resultados: cada uno de los elementos de la estructura modelada (corona, pilar, tornillo, implante, hueso cortical y esponjoso) sometida al incremento de esfuerzos, presentó valores von Mises y $\mu$ strain particulares con un comportamiento lineal. Al someter la estructura modelada a esfuerzos de $200 \mathrm{~N}$ y $800 \mathrm{~N}$, ninguno de los componentes sufrió deformaciones permanentes, es decir, no se superó el límite de fluencia.

Conclusión: de acuerdo al comportamiento mecánico de la estructura modelada en magnitudes de $800 \mathrm{~N}$, es posible la utilización de un implante dental en un incisivo central superior, debido a que las fuerzas parafuncionales generadas por el bruxismo no son superiores a las presentadas en la estructura modelada, en consecuencia, no generan deformaciones permanentes en el hueso.

Palabras clave: bruxismo, implantes dentales, análisis de elementos finitos.

\begin{abstract}
Introduction: The purpose of this research was to evaluate the bone microdeformation of bruxism with dental implants by means of the finite element analysis (FEA) method.

Materials and methods: One (1) Tapered Screw-Vent ${ }^{\circledR}$ implant (ref. TSVB10 Zimmer Dental): 13mm long $\mathrm{x}$ $3.7 \mathrm{~mm}$ diameter with a $3.5 \mathrm{~mm}$ platform, a Zirconium abutment, a screw, resin cement as the cementing agent, a monolithic ceramic crown of an upper central incisor, cortical bone and cancellous bone, was modeled using Solid Works 2010 (SolidWorks Corp., Concord, MA, USA), and later it was processed and analyzed with ANSYS version 14. The von Mises stresses and bone microdeformation (microstrain) were evaluated, applying oblique forces with magnitudes of $200 \mathrm{~N}$ and $800 \mathrm{~N}$. This analysis allowed for evaluating and comparing the microdeformation, both in cortical bone and in cancellous bone in two magnitudes $200 \mathrm{~N}$ and $800 \mathrm{~N}$.

Results: Each one of the elements of the modeled structure (crown, abutment, screw, implant, cortical and cancellous bone) when subjected to increased stress, presented particular von Mises and microstrain values with a linear behavior. By subjecting the modeled structure to forces of $200 \mathrm{~N}$ and $800 \mathrm{~N}$, none of the components suffered permanent deformation, that is, the yield point was not exceeded.

Conclusion: According to the mechanical behavior of the modeled structure in magnitudes of $800 \mathrm{~N}$, it is possible to use a dental implant in a maxillary central incisor, since the parafunctional forces generated by bruxism are not higher than those presented in the modeled structure; Consequently, they do not generate permanent bone deformations.
\end{abstract}

Keywords: bruxism, dental implants, finite element analysis. 
Ana María Sánchez Santamaría, Ana Catalina Castaño Posada, Federico Latorre Correa, Junes Abdul Villarraga Ossa, Juan Gustavo Diosa Peña, Samuel David Giraldo Gómez

\section{Resumo}

Introdução: O objetivo desta pesquisa foi avaliar a microdeformação óssea do bruxismo com implantes dentários por meio do método de análise de elementos finitos (FEA).

Materiais e métodos: Um (1) implante Tapered Screw-Vent ${ }^{\circledR}$ (ref. TSVB10 Zimmer Dental): 13 mm de comprimento x 3,7 mm de diâmetro com uma plataforma de 3,5 mm, um abutment de zircônio, um parafuso, cimento resinoso como agente de cimentação, um monolítico coroa de cerâmica de um incisivo central superior, osso cortical e osso esponjoso, foi modelada usando Solid Works 2010 (SolidWorks Corp., Concord, MA, EUA), e posteriormente processada e analisada com ANSYS versão 14. As tensões de von Mises e osso microdeformações (microstrain) foram avaliadas, aplicando forças oblíquas com magnitudes de $200 \mathrm{~N}$ e $800 \mathrm{~N}$. Essa análise permitiu avaliar e comparar a microdeformação, tanto no osso cortical quanto no osso esponjoso em duas magnitudes $200 \mathrm{~N}$ e $800 \mathrm{~N}$.

Resultados: Cada um dos elementos da estrutura modelada (coroa, pilar, parafuso, implante, osso cortical e esponjoso) quando submetido a tensões aumentadas, apresentou valores particulares de von Mises e micro-tensões com comportamento linear. Ao submeter a estrutura modelada a forças de $200 \mathrm{~N}$ e $800 \mathrm{~N}$, nenhum dos componentes sofreu deformação permanente, ou seja, o limite de escoamento não foi ultrapassado.

Conclusão: De acordo com o comportamento mecânico da estrutura modelada em magnitudes de $800 \mathrm{~N}$, é possível utilizar um implante dentário em um incisivo central superior, uma vez que as forças parafuncionais geradas pelo bruxismo não são superiores às apresentadas na estrutura modelada; Conseqüentemente, eles não geram deformações ósseas permanentes.

Palavras-chave: bruxismo, implantes dentários, análise de elementos finitos.

\section{Introducción}

Los procedimientos de implantología oral, en las últimas décadas, han experimentado un crecimiento significativo debido a sus ventajas mecánicas y biológicas sobre las opciones de tratamiento como prótesis parcial fija y prótesis parcial removible. A pesar de las altas tasas de éxito de los implantes dentales aún ocurren fallas, entre las cuales se encuentran problemas mecánicos relacionados con sobrecargas oclusales y esfuerzos (tensiles, compresivos, de corte), como ocurre en el caso del bruxismo (1).

Debido a que el bruxismo es un factor importante de sobre carga oclusal, se han generado diferentes posturas sobre la posibilidad de realizar un tratamiento implanto-soportado en pacientes bruxómanos. Estas van desde la contraindicación, sustentada en que las fuerzas a las que se encuentran sometidos los implantes dentales y sus estructuras por el bruxismo pueden causar una sobrecarga dando lugar a la pérdida de hueso alrededor de los implantes o incluso al fracaso de los mismos. También se afirma que las fuerzas parafuncionales ejercidas por bruxismo no se consideran un factor crítico en el éxito del implante y, por lo tanto, no afectan el tratamiento de rehabilitación $(2,3)$. 
Yang et al. (4), en su investigación, concluyen que los tejidos óseos reciben estímulos mecánicos y pueden sufrir cambios a favor o en contra. Por lo que durante la planificación de tratamientos de rehabilitación implanto-soportada, el bruxismo debe considerarse como un factor de riesgo, ya que las consecuencias de los movimientos parafuncionales pueden poner en peligro la longevidad de los implantes dentales, causar cambios en el hueso marginal y deterioro en la oseointegración.

Por otra parte, Raphael et al., (5) en su revisión de literatura concluyeron que aún no hay evidencia suficiente para aceptar o rechazar una relación causal entre el bruxismo y otras entidades. Es complejo definirlo como diagnóstico, comportamiento o factor de riesgo, lo cual sugiere la necesidad de diseñar nuevos estudios que permitan determinar la posible relación causa efecto entre el bruxismo, el fracaso de los implantes y la microdeformación ósea.

Debido a la falta de consenso en la literatura sobre la posibilidad de rehabilitar con implantes a pacientes bruxómanos, se dificulta la interpretación de la evidencia disponible en los estudios recientes. Esto también conlleva a una falta de claridad del clínico a la hora de planificar un tratamiento en este tipo de pacientes. Se evidencia una necesidad de estudios bien diseñados, para evaluar la microdeformación ósea en estos pacientes y su posible relación con una falla en el implante o en su oseointegración.

El propósito de este estudio fue analizar la microdeformación ósea con implantes dentales Zimmer en un central superior en pacientes con y sin bruxismo. Se utilizó como herramienta de simulación el análisis de elementos finitos para determinar el nivel de deformación ósea y, además, si este nivel de deformación represento un factor de riesgo en la rehabilitación con un implante dental.

\section{Materiales y métodos}

Se diseñó un modelo geométrico tridimensional utilizando el Software CAD de Solid Works 2010 (SolidWorks Corp., Concord, MA, USA). Se procesó y analizó a través del Software ANSYS versión 14. Como producto de estas dos operaciones se modelaron los siguientes componentes del sistema:

Un (1) implante Tapered Screw-Vent® (ref. TSVB10 zimmer- biomet. Warsaw, Indiana, USA) de $13 \mathrm{~mm}$. de longitud x $3.7 \mathrm{~mm}$ de diámetro con una plataforma de $3.5 \mathrm{~mm}$., de hexágono interno con su respectivo tornillo de fijación.

Un (1) pilar de zirconio Zimmer®, precontorneado, recto, con conexión interna hexagonal, perfil de emergencia de $4,5 \mathrm{~mm}$, altura del margen vestibular 1,0 $\mathrm{mm}$. 
Ana María Sánchez Santamaría, Ana Catalina Castaño Posada, Federico Latorre Correa, 5 Junes Abdul Villarraga Ossa, Juan Gustavo Diosa Peña, Samuel David Giraldo Gómez

Referencia número: ZRA341S, Casa Fabricante: Zimmer® Dental zimmer- biomet. Warsaw, Indiana, USA).

Cemento resinoso RelyX $\mathrm{X}^{\mathrm{TM}}$ Unicem 2 Automix. Casa fabricante: St. Paul, Minnesota,USA.

Una (1) corona cerámica monolítica en discilicato de litio (IPS E.max Prees), Casa fabricante: Ivoclar Vivadent, Schaan; Liechtenstein.

Dadas las condiciones del modelado se asumió una oseointegración del 100 \%.

Una vez obtenida la modelación, se realizó un estudio comparativo en el que se evaluaron las variables de esfuerzos máximos principales tensiles para el hueso cortical y hueso esponjoso, y esfuerzos mínimos principales compresivos (von Mises) para toda la estructura en cargas de $200 \mathrm{~N}$ y $800 \mathrm{~N}$, con un vector de fuerza oblicuo. Dicho análisis permitió valorar el comportamiento de las diferentes estructuras modeladas y los efectos generados en la interface hueso-implante.

Se escogió un implante para un incisivo central superior porque, según los reportes de la literatura, es el más utilizado por razones estéticas. El tipo de superficie del implante, el diámetro y la conexión del pilar, son los más utilizados para la rehabilitación oral en el sector anterior. Las restauraciones en esta zona están sometidas principalmente a cargas oblicuas con respecto a su eje central, generalmente en la cara lingual, tanto al realizar movimientos céntricos como excéntricos $(6,7)$.

\section{Modelado geométrico tridimensional:}

Todas las estructuras se modelaron de forma individual a partir de un diseño dibujado. Un implante para un incisivo central superior fue reproducido, simulando un hueso tipo DII, según la clasificación Lekholm y Zarb (8). Dicha sección de hueso se reprodujo a partir de un corte sagital extraído de una imagen tomográfica del maxilar superior, la cual incluía el hueso esponjoso y el cortical. Al modelar, no se tuvo en cuenta la encía porque no tenía importancia para la simulación de la microdeformación ósea. 

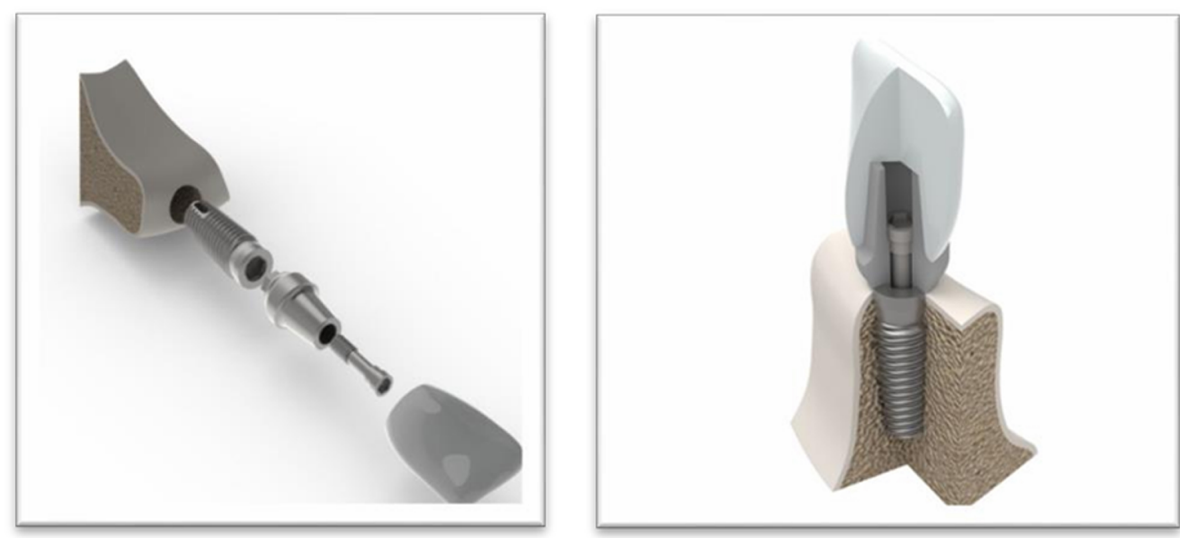

Figura 1. Imagen renderizada del hueso, implante, pilar, tornillo y corona Fuente: elaboración propia

Se evaluaron las propiedades isotrópicas para el implante, pilar, tornillo y corona, y las propiedades transversalmente isotrópicas para el hueso cortical y esponjoso. En los modelos óseos de los maxilares son introducidos una serie de valores que representan sus propiedades elásticas. Idealmente estas propiedades deben representar la anisotropía del hueso. El hueso es considerado anisotrópico porque muestra distintas propiedades mecánicas dependiendo de la dirección desde la cual sean medidas. Sin embargo, por la complejidad de la modelación de una estructura con dichas características, puede ser aplicable una aproximación debido a que el módulo elástico del hueso cortical en direcciones buco lingual y supero inferior no son significativamente diferentes, e igualmente este módulo en el hueso es similar en direcciones bucolingual y mesiodistal. Con esta aproximación o isotropía transversal (ortotrópico) que se compone de cinco propiedades elásticas independientes se representa de la mejor forma la anisotropía del hueso (9). Las propiedades mecánicas de los materiales usados se muestran en la tabla 1.

Tabla 1. Propiedades mecánicas de las estructuras y materiales modelados

\begin{tabular}{lccccccc}
\hline \multirow{2}{*}{ MATERIAL } & \multicolumn{6}{c}{ E Limite elástico (MPa) } & \multicolumn{2}{c}{ V Coeficiente de Poisson } & \multicolumn{2}{c}{ Límite de } \\
\cline { 2 - 6 } & $\mathbf{X}$ & $\mathbf{Y}$ & $\mathbf{Z}$ & $\mathbf{X X}$ & $\mathbf{X Y}$ & $\mathbf{X Z}$ & fluencia (MPa) \\
\hline Hueso cortical $(9,10,11)$ & 10.000 & 11.000 & 14.300 & .370 & .330 & .230 & $60-120$ \\
\hline $\begin{array}{l}\text { Hueso esponjoso } \\
(9,10,11)\end{array}$ & 1148 & 210 & 1148 & .50 & .320 & .10 & $60-120$ \\
\hline $\begin{array}{l}\text { Implante de titanio } \\
(12,13,14)\end{array}$ & 110.000 & 110.000 & 110.000 & .330 & .330 & .330 & 800 \\
\hline
\end{tabular}


Ana María Sánchez Santamaría, Ana Catalina Castaño Posada, Federico Latorre Correa, 7 Junes Abdul Villarraga Ossa, Juan Gustavo Diosa Peña, Samuel David Giraldo Gómez

(viene)

\begin{tabular}{|c|c|c|c|c|c|c|c|}
\hline \multirow{2}{*}{ MATERIAL } & \multicolumn{3}{|c|}{ E Limite elástico (MPa) } & \multicolumn{3}{|c|}{ V Coeficiente de Poisson } & \multirow{2}{*}{$\begin{array}{c}\text { Límite de } \\
\text { fluencia (MPa) }\end{array}$} \\
\hline & $x$ & $\mathbf{Y}$ & $\mathbf{Z}$ & $x X$ & $\mathbf{X Y}$ & $X Z$ & \\
\hline $\begin{array}{l}\text { Tornillo del pilar titanio } \\
(12,13,14)\end{array}$ & 110.000 & 110.000 & 110.000 & .330 & .330 & .330 & 800 \\
\hline $\begin{array}{l}\text { Pilar de Circona } \\
(15,16,17)\end{array}$ & 200.000 & 200.000 & 200.000 & .270 & .270 & .270 & 900 \\
\hline Cemento resinoso (18) & 6600 & 6600 & 6600 & .300 & .300 & .300 & 48 \\
\hline $\begin{array}{l}\text { Corona Cerámica } \\
\text { Monolítica en disilicato } \\
\text { de litio (19) }\end{array}$ & 95.000 & 95.000 & 95.000 & .23 & .23 & .23 & 350 \\
\hline
\end{tabular}

Fuente: elaboración propia

Para el análisis del comportamiento mecánico de la estructura se realizó un análisis elástico lineal donde se utilizaron elementos tetraédricos (elementos que permiten tres grados de libertad traslacional y tres rotacionales, por nodo) con el fin de obtener una mejor aproximación de las geometrías de las partes. De esta manera se obtuvo una malla tridimensional de elementos finitos de los componentes del modelo.

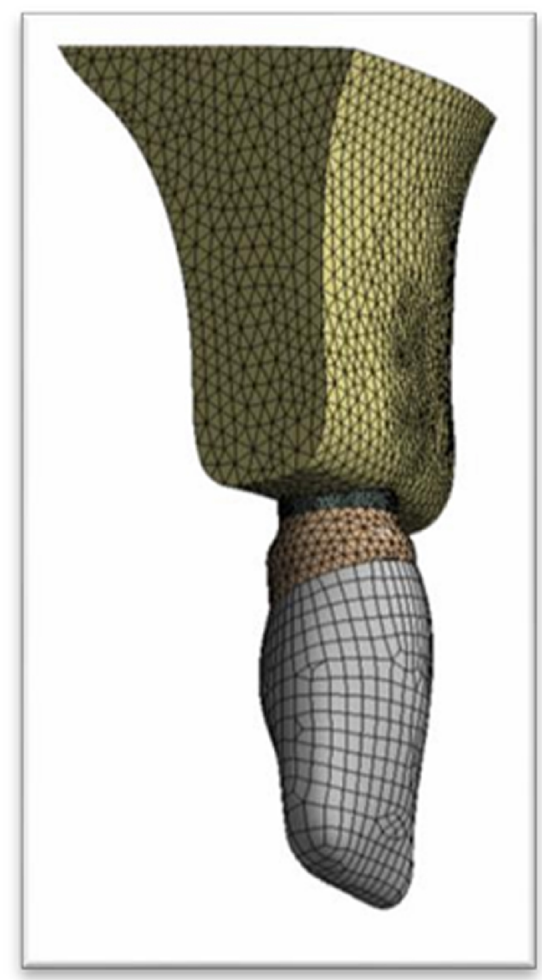

Figura 2. Malla sólida tetraédrica de las estructuras Fuente: elaboración propia 
8 Simulación de la microdeformación ósea de bruxismo en implante dental anterior

Tabla 2. Detalles de la malla de los modelos estudiados

\begin{tabular}{lcc}
\hline \multicolumn{1}{c}{ DESCRIPCIÓN } & CANTIDAD NODOS & CANDIDAD ELEMENTOS \\
\hline Implante & 730514 & 501246 \\
\hline Pilar & 1244044 & 896129 \\
\hline Tornillo & 21270 & 11912 \\
\hline Corona & 251476 & 71686 \\
\hline Cortical & 1443978 & 1031854 \\
\hline Esponjoso & 1051952 & 7746127 \\
\hline
\end{tabular}

Fuente: elaboración propia

Este modelo tuvo un alto número de elementos por que se modelaron varios componentes y porque se pretendía tener un buen valor de la calidad promedio de los elementos.

\section{Condiciones de la carga:}

Se realizó una condición de precarga en el tornillo, empleando la siguiente formulación:

$T=k * F * d$

T: torque, k:0.2 F: fuerza axial d: diámetro del tornillo

El torque aplicado es de $30 \mathrm{~N}$ cm según lo recomendado por la casa fabricante ${ }^{9}$. Esta fuerza axial se relaciona a las ecuaciones:

$\mathrm{DL}=(\mathrm{P} * \mathrm{~L}) /(\mathrm{A} \star \mathrm{E})$

$D L=a * D T * L$ Siendo

DL: cambio de longitud P: Fuerza axial

A: área del tornillo

E: módulo de elasticidad

a: coeficiente de dilatación térmica DT: Cambio de temperatura

Esa contracción térmica uniaxial hace las veces de la precarga en el tornillo y la fuerza resultante es la equivalente aplicada por el torque. La carga estática se realizó sometiendo al modelo a una fuerza de 45 grados a nivel de la pared lingual. Ver figura 3. 
Ana María Sánchez Santamaría, Ana Catalina Castaño Posada, Federico Latorre Correa, Junes Abdul Villarraga Ossa, Juan Gustavo Diosa Peña, Samuel David Giraldo Gómez

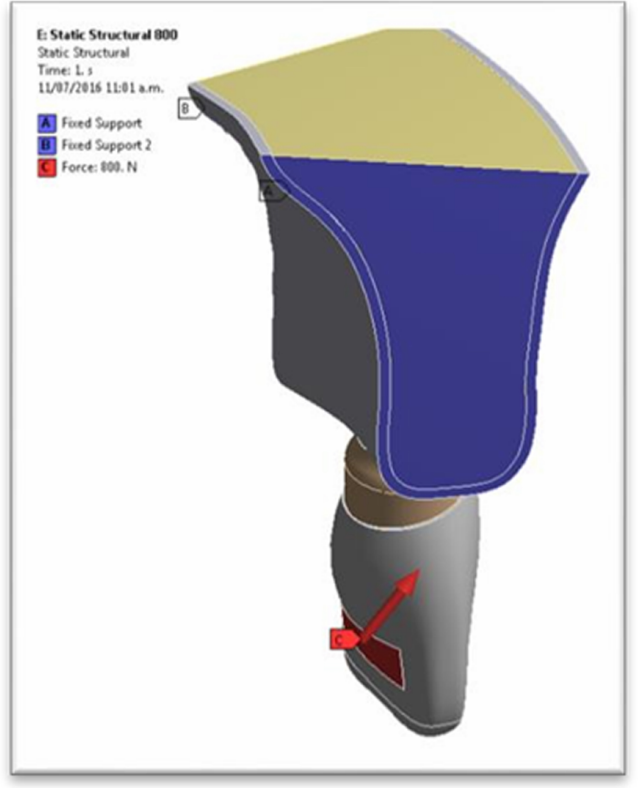

Figura 3. Dirección y ubicación de las cargas (áreas en rojo) en el modelo Fuente: elaboración propia

Al validar la malla, se utilizó el método adaptativo conocido como método $\mathrm{H}$, el cual consiste en realizar un refinamiento en el tamaño de la malla en los lugares de mayor interés para el estudio.

\section{Resultados}

El reporte de los resultados se hizo de manera descriptiva y se adicionó la gráfica correspondiente a los esfuerzos von Mises de toda la estructura y $\mu$ strain para el hueso cortical y esponjoso, en el análisis comparativo de la distribución de los esfuerzos se evaluaron cargas de $200 \mathrm{~N}$ y $800 \mathrm{~N}$. Las siguientes figuras muestran los valores de los esfuerzos para cada componente. 


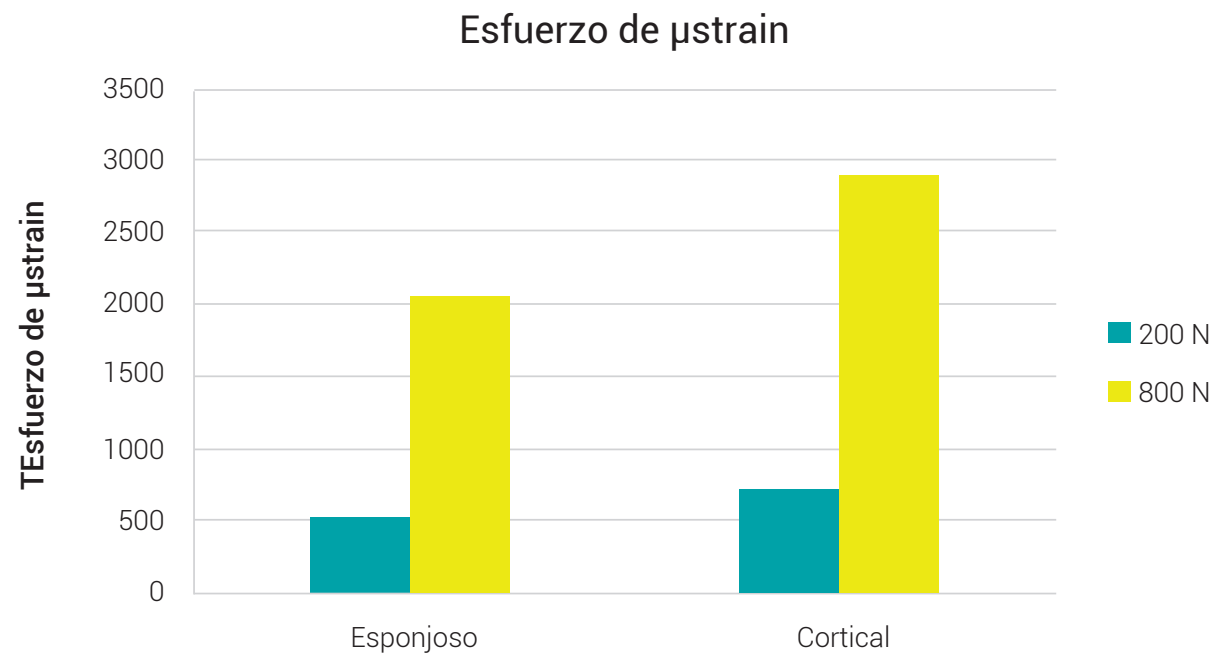

Figura 4. Comparativo. Esfuerzos $\mu$ strain a $200 \mathrm{~N}$ y $800 \mathrm{~N}$ del hueso cortical y hueso esponjoso

Fuente: elaboración propia

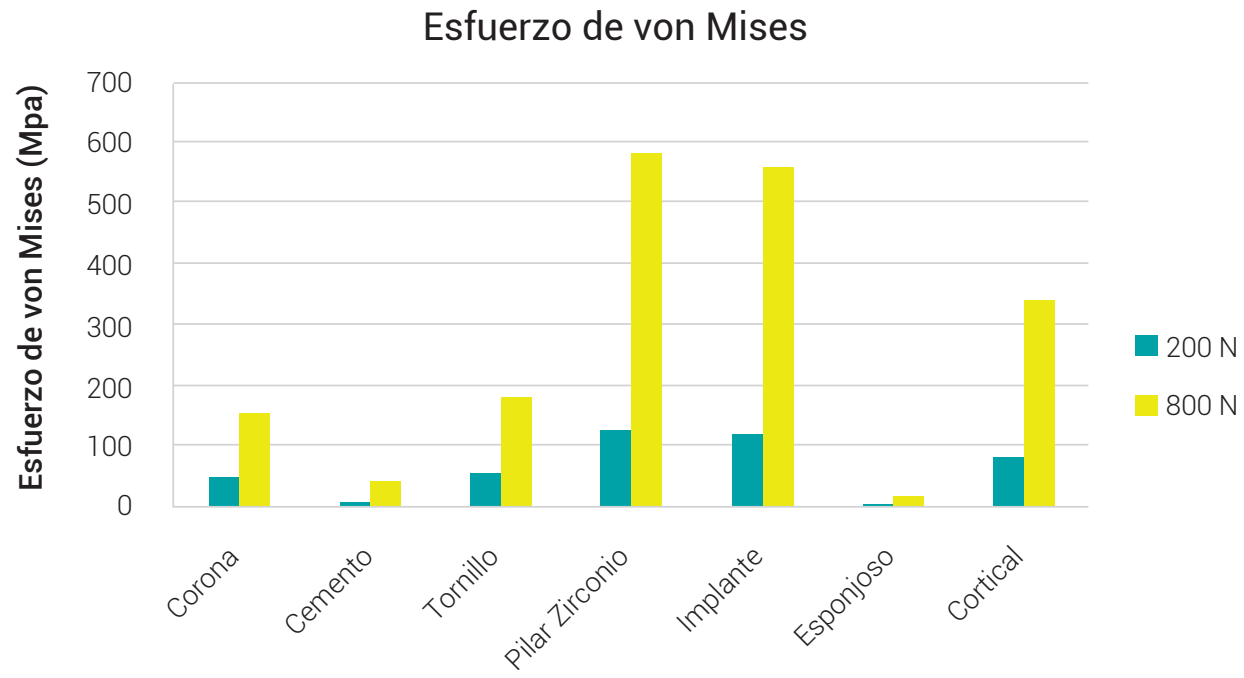

Figura 5. Comparativo. Esfuerzos von Mises a $200 \mathrm{~N}$ y $800 \mathrm{~N}$ de los elementos del modelo. Fuente: elaboración propia

\section{Distribución de esfuerzos en la corona:}

El esfuerzo von Mises para la corona aumentó linealmente en una relación de 1 a 3 al incrementar la fuerza de $200 \mathrm{~N}$ a $800 \mathrm{~N}$, mostrando un valor máximo de esfuerzo von Mises de 156,7 MPa, valor que no excede el límite de fluencia, por tanto, el elemento 
no presentó una deformación permanente. La concentración de esfuerzos se encontró en la zona lingual y cervical.

\section{Distribución de esfuerzos en el cemento:}

El esfuerzo von Mises para el cemento aumentó linealmente en una relación de 1 a 3 al incrementar la fuerza de $200 \mathrm{~N}$ a $800 \mathrm{~N}$, mostrando un valor máximo de esfuerzo von Mises de 42,8 MPa, valor que no excede el límite de fluencia, por tanto el elemento no presentó una deformación permanente. La concentración de esfuerzos se encontró en la porción cervical.

\section{Distribución de esfuerzos en el Pilar de Zirconio:}

El esfuerzo von Mises para el pilar de zirconio aumentó linealmente en una relación de 1 a 4,6 al incrementar la fuerza de $200 \mathrm{~N}$ a $800 \mathrm{~N}$, mostrando un valor máximo de esfuerzo von Mises de $584 \mathrm{MPa}$, a pesar de que fue el componente de la modelación que presentó el mayor valor von Mises, no se excedió el límite de fluencia, por tanto, el elemento no presento una deformación permanente. La concentración de esfuerzos se encontró en la parte cervical del pilar en su parte interna.

\section{Distribución de esfuerzos en el tornillo:}

El esfuerzo von Mises para el tornillo aumentó linealmente en una relación de 1 a 3 al incrementar la fuerza de $200 \mathrm{~N}$ a $800 \mathrm{~N}$, mostrando un valor máximo de esfuerzo von Mises de $181 \mathrm{MPa}$, valor que no excede el límite de fluencia, por tanto, el elemento no presentó una deformación permanente. La concentración de los esfuerzos en el tornillo estuvo ubicada en la superficie externa de las roscas del tornillo.

\section{Distribución de esfuerzos en el implante:}

El esfuerzo von Mises para el implante aumentó linealmente en una relación de 1 a 4,5 al incrementar la fuerza de $200 \mathrm{~N}$ a $800 \mathrm{~N}$, mostrando un valor máximo de esfuerzo von Mises de $561 \mathrm{MPa}$, después del pilar, el implante es el segundo componente de la estructura que muestra un mayor valor von Mises, sin embargo, el valor no alcanza a exceder el límite de fluencia, por tanto el elemento no presentó una deformación permanente. La concentración de esfuerzos se localizó en la zona superior de la plataforma del implante. 


\section{Distribución de esfuerzos en las estructuras biológicas:}

El valor $\mu$ strain mostró un aumento lineal para el hueso cortical en una relación de 1 a 5 al incrementar la fuerza de $200 \mathrm{~N}$ a $800 \mathrm{~N}$, mostrando un valor máximo $\mu$ strain de 2890, mientras que el hueso esponjoso arrojó un aumento lineal en una relación de 1 a 4 al someterlo a las mismas fuerzas, registrado un valor máximo $\mu$ strain de 2050, la aplicación de cargas estáticas arrojó valores que no alcanzaron a superar los límites elásticos de dichos tejidos y no provocaron deformaciones permanentes.

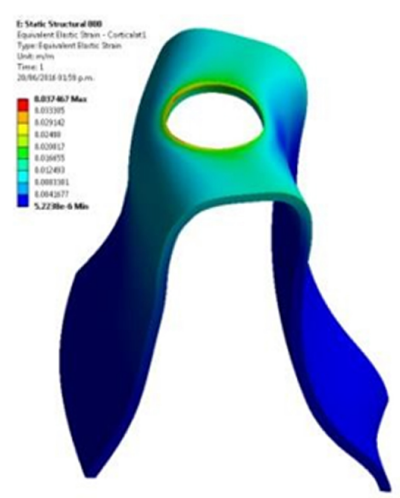

A) Hueso cortical

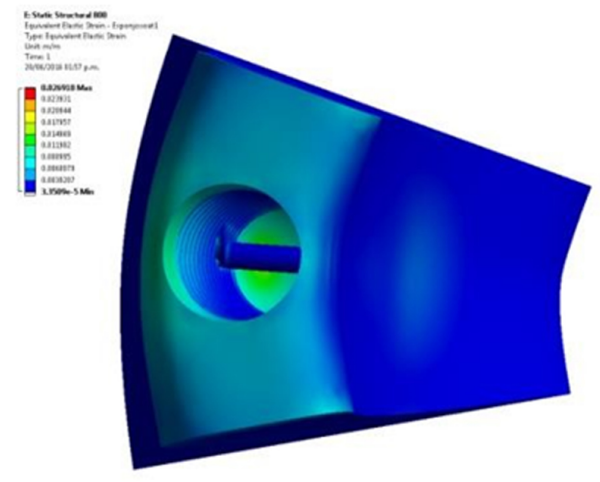

B) Hueso esponjoso

Figura 6. Áreas de concentración de los esfuerzos $\mu$ strain en el hueso cortical (a) y hueso esponjoso (b), bajo una carga de $800 \mathrm{~N}$

Fuente: elaboración propia

El resultado von Mises para el hueso cortical aumento linealmente en una relación de 1 a 4, al incrementar la fuerza de $200 \mathrm{~N}$ a $800 \mathrm{~N}$, mostrando un valor máximo de esfuerzo von Mises de $341 \mathrm{MPa}$, después del pilar y el implante, es el tercer componente de la estructura que muestra un mayor valor von Mises. El esfuerzo von Mises para el hueso esponjoso aumentó linealmente en una relación de 1 a 3.8, al incrementar la fuerza de $200 \mathrm{~N}$ a $800 \mathrm{~N}$, mostrando un valor máximo de esfuerzo von Mises de 18,6 MPa, siendo este el valor más bajo registrado por toda la estructura modelada.

\section{Discusión}

En la actualidad, el bruxismo es considerado una preocupación o incluso una contraindicación para el tratamiento con implantes dentales (20-22). Hsu y colaboradores et al (23), reportaron una relación significativa entre el bruxismo o sobrecarga oclusal 
Ana María Sánchez Santamaría, Ana Catalina Castaño Posada, Federico Latorre Correa, 13 Junes Abdul Villarraga Ossa, Juan Gustavo Diosa Peña, Samuel David Giraldo Gómez

como principal factor etiológico, produciéndose complicaciones biomecánicas que incluyeron pérdida marginal ósea, fractura de materiales, pérdida o aflojamiento de tornillos y hasta el fracaso del implante. Glauser et al. (24) encontraron un mayor porcentaje de pérdida del implante en pacientes bruxómanos que en pacientes no bruxómanos después de un año (41 \% vs 12 \%). Bragger et al. (25) reportaron problemas biomecánicos en los implantes de pacientes bruxómanos en el 60 \% de los casos, comparado con cerca del $20 \%$ de problemas en los no bruxómanos.

Por otro lado, algunos estudios no reportan asociaciones significativas entre el bruxismo y problemas con los implantes dentales. De acuerdo a Manfredini et al (26), la evidencia que justifica la contraindicación de los implantes dentales en pacientes bruxómanos, está basada únicamente en la experiencia clínica, ya que los estudios que establecen una relación de causa y efecto entre el bruxismo y daño periodontal con posible fracaso de los implantes, no presentan resultados concluyentes ni específicos en la literatura.

Para el presente estudio se realizó un análisis comparativo, en el que se evaluaron las variables $\mu$ strain y esfuerzos von Mises en cargas de $200 \mathrm{~N}$ y $800 \mathrm{~N}$ respectivamente, con un vector de fuerza oblicuo. La carga inicial de $200 \mathrm{~N}$ corresponde a la carga oclusal normal reportada por la literatura para el sector anterior en un incisivo central superior $(11,14)$, así mismo, en la modelación se estableció un límite máximo de $800 \mathrm{~N}$ como factor de seguridad puesto que, según los estudios realizados a la fecha, la máxima carga oclusal registrada en pacientes bruxómanos no supera los (682N), dejando así un amplio margen de error llegado el caso de superarse este tope (15).

De acuerdo a los resultados obtenidos en este estudio, el hueso cortical mostró un mayor valor von Mises (341 MPa) debido a su rigidez y menor deformación con respecto al tejido óseo esponjoso, mientras que el hueso esponjoso mostró el valor von Mises más bajo (18,6 MPa) debido a su bajo módulo de elasticidad, lo que lleva a resistir fuerzas de compresión y tensión, así como de absorber y transmitir a las otras estructuras los efectos de las cargas repetitivas (27). Este resultado se corrobora en el estudio de Kurniawan et al. (11), donde concluyen que, a menor densidad ósea, mayor será el esfuerzo inducido en el hueso cortical y menor valor se distribuye en el hueso esponjoso.

Teniendo en cuenta los resultados $\mu$ strain y los niveles de microdeformación $[\mu \varepsilon]$ sistematizados por Frost (28), ver (Tabla 3) tanto el hueso cortical como el hueso esponjoso, bajo la carga de $800 \mathrm{~N}$ de este estudio, presentaron un nivel de microdeformación denominado "Sobre Carga Ligera", lo cual significa que es posible que se generen fracturas en el hueso por fatiga, pero el tejido por sí solo inicia una reparación tisular, aumentando el volumen óseo para reducir los esfuerzos. El autor 
afırma además que se requiere de una cierta cantidad de esfuerzo para mantener la homeostasis ósea. Una estimulación muy pequeña resulta en atrofia del hueso con la inducción de microfracturas y pérdida del tejido óseo. Estos resultados se asemejan con los encontrados en el estudio a 3 años de Luo Q et al. (29) en donde concluyeron que falta soporte científico para asegurar la pérdida ósea por sobrecarga y los contactos cambian con el tiempo. En el estudio de Greenstein $\mathrm{G}$ et al. (30), reportan que si una carga se encuentra por debajo del límite destructivo del hueso, puede ser un estímulo e inducir aposición y aumentar la densidad ósea. La aposición ósea es un mecanismo importante de compensación cuando la tensión supera su rango fisiológico. Es decir, el estrés y la tensión inducen un proceso reparativo, debido a que el hueso se vuelve más fuerte.

Tabla 3. Niveles de Microdeformación [ $\mu \varepsilon]$ de Frost (19)

\begin{tabular}{ccccc}
\hline Inactividad ósea & $\begin{array}{c}\text { Zona de } \\
\text { adaptación }\end{array}$ & $\begin{array}{c}\text { Sobrecarga } \\
\text { ligera }\end{array}$ & $\begin{array}{c}\text { Sobrecarga } \\
\text { patológica }\end{array}$ & $\begin{array}{c}\text { Fractura } \\
\text { espontanea }\end{array}$ \\
\hline $0-50$ & $50-150$ & $150-3000$ & $3.000-10.000$ & $10.000-20.000$ \\
\hline
\end{tabular}

Fuente: elaboración propia

Se puede observar en este estudio, que los esfuerzos máximos von Mises de todo el sistema, tanto en cargas de $200 \mathrm{~N}$ como en las de $800 \mathrm{~N}$ se presentan principalmente en el pilar y en el cuerpo del implante, que son los elementos con el módulo de elasticidad más alto. Sin embargo, los valores obtenidos no superaron el límite de fluencia de los materiales. Esto concuerda con lo encontrado en estudios como el de Roldán F y cols (6), donde el pilar, el tornillo y el implante fueron los elementos que presentaron un mayor valor von Mises durante su estudio.

Estudios como el de Radaelli et al. (31) encontraron que las fuerzas parafuncionales aumentan los esfuerzos en todas las estructuras, ellos argumentan que con dispositivos oclusales se distribuye y se protege el sistema, sin embargo, nuestro estudio sugiere que aun sin estos dispositivos, se mantienen las fuerzas en un rango tolerable para las estructuras biológicas y mecánicas.

Basándose en los resultados del presente estudio y considerando las limitaciones asociadas a la modelación tridimensional con características óseas específicas, una oseointegración del 100 \% y una fuerza estática, se considera que las cargas alrededor de $800 \mathrm{~N}$ no afectan de manera significativa los diferentes tipos de hueso, ni a los componentes protésicos, estos resultados coinciden con los encontrados por 
Ana María Sánchez Santamaría, Ana Catalina Castaño Posada, Federico Latorre Correa, 15 Junes Abdul Villarraga Ossa, Juan Gustavo Diosa Peña, Samuel David Giraldo Gómez

Manfredini (26), en donde no se encontró una causa directa entre el bruxismo, pérdida ósea marginal y posible falla de los implantes.

Se debe considerar que este estudio es una simulación, por lo que sus resultados son una información importante, pero se debe tener precaución al extrapolar a la clínica y se debe hacer su valoración con estudios clínicos similares.

\section{Conclusiones}

De acuerdo al comportamiento mecánico de la estructura modelada, en la carga de $800 \mathrm{~N}$ es adecuado la utilización de un implante dental en un incisivo central superior, debido a que las fuerzas parafuncionales generadas por el bruxismo no son superiores a las presentadas en la estructura modelada, en consecuencia, no generan deformaciones permanentes en el hueso, como se deriva de los resultados obtenidos.

Al someter la estructura modelada a un incremento de fuerza de $200 \mathrm{~N}$ a $800 \mathrm{~N}$ cada componente modelado presenta valores von Mises y $\mu$ strain particulares, lo que indica que cada componente reacciona de manera diferente al aplicarle cargas.

La microdeformación presentada en el hueso cortical y esponjoso, corresponden a una "Sobre Carga Ligera", la cual, no representa un riesgo en el tratamiento según este estudio en cargas de $800 \mathrm{~N}$ con un implante dental para un central superior.

No se generaron esfuerzos patológicos para ninguna de las estructuras, sin embargo, no están simuladas otras variables como fatiga, envejecimiento y otras causas que pueden determinar comportamientos a largo plazo de las estructuras y tejidos biológicos.

\section{Referencias}

1. Díaz Sadowsky SJ. Occlusal overload with dental implants: a review. Int J Implant Dent. 2019; 23; 5(1):29. doi: 10.1186/s40729-019-0180-8.

2. Stoichkov B,Kirov D. Analysis of the causes of dental implant fracture: A retrospective clinical study. Quintessence Int. 2018; 49(4):279-286. doi: 10.3290/j.qi.a39846.

3. Lobezoo F, Ahlberg J, Mandredini D, Winocur E. Are bruxism and the bite causally? J Oral Rehabil. 2012; 39: 489-501. doi: 10.1111/j.1365-2842.2012.02298.x. 
4. Yang H, Xu X, Bullock W, Main P R. Adaptive changes in micromechanical environments of cancellous and cortical bone in response to in vivo loading and disuse. J biomech. 2019; 24(89): 85-94. doi: 10.1016/j.jbiomech.2019.04.021

5. Raphael JK, Santiago V, Lobbezoo F. Is bruxism a disorder or a behavior? Rethinking the international consensus on defining and grading of bruxism. J Oral Rehabil. 2016; 43(10): 791-8. doi: 10.1111/joor.12413

6. Roldán VFM, Escobar RJC, Latorre CF. Análisis lineal y no lineal de los esfuerzos en pilares de circonio preformados. Elementos finitos. Rev. Ing.Biomed. 2014; 8 (16):39-50. doi. org/10.24050/19099762.n16.2014.651

7. Kim K-S, Lim Y-J.Axial displacementand removal torque changes of five differentimplant-abutment connections under static vertical loading. Materials (basel). 2020;4(13):699. doi: 10.3390/ma13030699.

8. Misch C. Bone density: A key determinant for treatment planning. Part I: diagnosis and rationale. In: Contemporary Implant Dentistry. $3^{\text {a }}$ ed. Ed Mosby, Elsevier, Canada 2009. pp.3-232

9. Kurniawan D. Finite element analysis of bone-implant biomechanics: refinement throughfeaturing various osseointegration conditions. Int J Oral Maxillofac Surg. 2012; 41(9): 10901096. doi: 10.1016/j.ijom.2011.12.026.

10. O’Mahony AM, Williams JL, Spencer P. Anisotropic elasticity of cortical and cancellous bone in the posterior mandible increases peri-implant stress under oblique loading. Clin. Oral Impl Res, 12, 648-657, 2001. doi: 10.1034/j.1600-0501.2001.120614.x

11. Rodríguez-Lopez MA, Giraldo-Gómez SD, Latorre-Correa F, Villarraga-Ossa JA, Diosa-Peña JG. Evaluación de la distribución de esfuerzos de una restauración inmediata implantosoportada en tres fases de cicatrización. Análisis de elementos finitos. Rev Nac Odontol. 2019; 15(28): 1-15. doi.org/10.16925/2357-4607.2019.01.06.

12. Tapered screw-vent implant system-product catalog. Zimmer Dental 2009. p. 1-86.

13. Zhang L-C,Chen L-Y. A review on biomedical titanium alloys: recent progress and prospect. Adv.Eng.Mater. 2019, 21: 1-29. doi.org/10.1002/adem.201801215.

14. Aguilar JH, Latorre CF, Junes Abdul Villarraga JA. Evaluación de la micro-deformación de la zona maxilar anterior con regeneración. Análisis de elementos finitos. Odontol. Sanmarquina 2019; 22(2): 110-117. doi: org/10.15381/os.v22i2.16223. 
Ana María Sánchez Santamaría, Ana Catalina Castaño Posada, Federico Latorre Correa, Junes Abdul Villarraga Ossa, Juan Gustavo Diosa Peña, Samuel David Giraldo Gómez

15. Suárez NT, Escobar JC, Latorre F, Villarraga J. Comportamiento estático de un pilar de circona sometido a envejecimiento artificial. Método de elementos finitos. Rev Fac Odontol Univ Antioq 2015;27(1): 62-94. doi: dx.doi.org/10.17533/udea.rfo.v27n1a2

16. Won Joo. Zimmer ${ }^{\circledR}$ Contour Ceramic Abutments. Precision engineered for strength, esthetics and clinical versatility. USA: Zimmer Dental Inc.; 2008. 1-6.

17. Okada M, Taketa H, Satoshi HE, Ikie M, Matsumoto T. Improvement of mechanical properties of Y-TZP by thermal annealing with monoclinic zirconia nanoparticle coating. Dent Mater. 2019; 35(7): 970-978. doi: 10.1016/j.dental.2019.04.002

18. 3M. Technical Data Sheet.RelyX TM Unicem 2 Automix. Recuperado de: http://multimedia.3m.com/mws/mediawebserver?mwsld=66666UuZjcFSLXTtnxf218TXEVuQEcuZgVs6EVs6 E666666--\&fn=rx_u2_auto_tds.pdf.

19. IPS e-maxPress. ScientificDocumentation. Recuperado de: http://www.ivoclarvivadent.co/ es-es/productos/ceramica-sin-metal/ips-emax-system-odontologo/ips-emax-disilicato -de-litio

20. Pjetursson BE, Heimisdottir K. Dental implants - are they better than natural teeth? Eur J Oral Sci. 2018 Oct; 126 Suppl 1:81-87. doi: 10.1111/eos.12543.

21. Koyano K, Esaki D. Occlusion on oral implants: current clinical guidelines. J Oral Rehabil. 2015; 42(2): 153-61. doi: 10.1111/joor.12239.

22. Sarmento HR, Dantas RV, Pereira-Cenci T, Faot F. Elements of implant-supported rehabilitation planning in patients with bruxism. J Craniofac Surg. 2012; 23(6): 1905-9. doi: 10.1097/ SCS.0b013e31826b8267

23. Hsu Y-T, Fu J-H, Al-Hezaimir K, Wang H-L. Biomechanical implant complications: a systematic review of clinical studies of implants with at least 1 year of functional loading. Int J Oral Maxillofac Implants. 2012; 27(4): 894-904.

24. Glauser R, Rée A, Lundgren A, Gottlow J, Hämmerle CH, Schärer P. Immediate occlusal loading of Brånemark implants applied in various jawbone regions: a prospective, 1-year clinical study. Clin Implant Dent Relat Res. 2001; 3(4): 204-13. doi: 10.1111/j.1708-8208.2001. tb00142.x. 
25. Brägger U, Aeschlimann S, Bürgin W, Hämmerle CH, Lang NP. Biological and technical complications and failures with fixed partial dentures (FPD) on implants and teeth afterfourto fiveyears of function. Clin Oral Implant Res. 2001; 12: 26- 34. doi: 10.1034/j.1600-0501.2001.012001026.

26. Manfredini D, Ahlberg J, Mura R, Lobbezoo F. Bruxism is unlikely to cause damage to the periodontium: findings from a systematic literature assessment. J Periodontol. 2015; 86(4): 546-55. doi: 10.1902/jop.2014.140539.

27. Roberts WE. Bone physiology, metabolism, and biomechanics. En: Misch CE. Contemporary Implant Dentistry. 3rd ed. St. Louis: Mosby; 2008. pp.557-598.

28. Frost HM. A 2003 update of bone physiology and Wolff 's Law for clinicians. Angle Orthod 2004;74(1):3-15. doi: 10.1043/0003-3219(2004)074<0003:AUOBPA>2.0.CO;2.

29. Luo Q, Ding Q, Zhang L. Xie Q. Analyzing the occlusion variation of single posterior implantsupported fixed prostheses by using the T-scan system: A prospective 3-year follow-up study. J Prosthet Dent. 2020; 123(1): 79-84. doi: 10.1016/j.prosdent.2018.12.012

30. Gary Greenstein, DDS, MS; John Cavallaro, DDS; Dennis Tarnow, DDS, Assessing bone's adaptive capacity around dental implants, J am Dent Assoc. 2013; 144(4): 362-368. doi: 10.14219/ jada.archive.2013.0129.

31. Radaelli B.M.T, Idogana TH, Spazzin OA, Noritomi YP, Bóscato N. Parafunctional loading and occlusal device on stress distribution around implants: a 3D finite element analysis. J Prosthet Dent. 2018; 120(4): 565-572. doi: 10.1016/j.prosdent.2017.12.023. 